\title{
Biomarkers of Attenuation in the Leishmania donovani Centrin Gene Deleted Cell Line-Requirements for Safety in a Live Vaccine Candidate
}

\author{
Robert Duncan, ${ }^{*, a}$, Ranadhir Dey ${ }^{\mathrm{a}}$, Keiko Tomioka ${ }^{\mathrm{a}, \mathrm{b}}$, Heather Hairston ${ }^{\mathrm{a}, \mathrm{c}}$, Angamuthu Selvapandi- \\ yan $^{\mathrm{a}, \mathrm{d}}$ and Hira L. Nakhasi ${ }^{\mathrm{a}}$ \\ ${ }^{a}$ Division of Emerging and Transfusion Transmitted Diseases, Office of Blood Research and Review, Center for \\ Biologics Evaluation and Research, Food and Drug Administration, Bethesda, MD 20892, USA \\ ${ }^{b}$ Hepatitis Viruses Section, LID, NIAID, Rockville, MD 20852, USA \\ 'Infectious Disease Clinical Research Program, National Naval Medical Center, Bethesda, MD 20889, USA \\ ${ }^{d}$ Institute of Molecular Medicine, 254 Okhla Industrial Estate, Phase III, New Delhi,11002a, India
}

\begin{abstract}
Biomarkers of the attenuated phenotype are needed to develop live attenuated parasites into safe Leishmania vaccines. The centrin-1 gene deleted strain of Leishmania donovani $\left(\right.$ LdCEN1 $\left.{ }^{-/}\right)$shows promise as a vaccine candidate. To identify genes whose expression patterns are indicators of attenuation, the $L d C E N 1^{-/-}$line was compared to wild type by gene expression microarray. Two genes, one coding for a $27 \mathrm{kDa}$ protein (p27) and another coding for putative Argininosuccinate Synthase (AS) have such expression patterns. Both genes express a higher RNA level in the amastigote stage than in the promastigote stage of wild type cells; however they are down-regulated in the $L d C E N 1^{-/}$amastigote cells. Western blots indicated that the AS protein level is also reduced in the $L d C E N 1^{-/}$amastigotes, while the p27 protein level is not reduced even when its mRNA level has diminished. Northern and Western blot analysis with these two biomarkers showed that $L d C E N 1^{-/}$parasites recovered after five weeks of infection in mice had the same expression pattern as they had prior to infection and episomal expression of centrin in the $L d C E N 1^{-/}$cells restored normal expression of both genes. Measurement of the expression of these two genes in infected macrophages by RT-PCR indicated the same pattern as in cultured cells. Therefore, both the mRNA and/or the protein levels of these two genes could be used as biomarkers of attenuation to monitor the safety of the $L d C E N 1^{-/}$cell line as it is developed as a potential vaccine.
\end{abstract}

Keywords: Leishmaniasis vaccines, Parasites, Vaccines, Live attenuated, Gene expression, Attenuated phentype biomarkers.

\section{INTRODUCTION}

Leishmania donovani is a protozoan parasite that is the causative agent of visceral leishmaniasis, also known as kala-azar. This fatal disease is currently a serious problem in the eastern part of India, Bangaladesh and parts of Sudan. Treatments are available, but are costly, may require hospitalization, and resistant strains of the parasite have rendered many traditional drugs ineffective [1].

A vaccine to prevent leishmaniasis has been a goal for nearly a century because a cured infection protects the individual from reinfection. Over many years numerous approaches have been attempted such as whole killed parasites, addition of adjuvants such as Bacillus Calmette-Guerin [2], recombinant protein antigens either mixed with appropriate adjuvants for injection or expressed by attenuated bacterial or viral vectors [3-4], and DNA vaccines [5-7]. Though some recombinant antigen vaccines are still under investigation, none are ready for routine clinical use [8-9]. The ineffectiveness of subunit based strategies and our improved

*Address correspondence to this author at the Division of Emerging and Transfusion Transmitted Diseases, Office of Blood Research and Review, Center for Biologics Evaluation and Research, Food and Drug Administration, Bethesda, 1401 Rockville Pk. HFM310, MD 20892, USA; Tel: 301496-1651; Fax: 301-480-7928; E-mail: Robert.Duncan@fda.hhs.gov understanding of the immune response to Leishmania suggest a live attenuated vaccine that would expose the recipient to complex antigens in the right context could be an alternate approach as a vaccine for leishmaniasis [10].

We have developed an attenuated line of Leishmania donovani by targeted deletion of the centrin 1 gene [11]. Centrins are highly conserved calcium binding proteins that are associated with the centrosome of all eukaryotes examined to date. Centrin 1 is required for centrosome duplication and segregation and its absence results in the failure of appropriate cell division [12]. The centrin 1-deleted L. donovani cells grow normally as promastigotes, the stage in the parasite life cycle normally found in the insect vector. However, when they infect host macrophage cells and differentiate into the amastigote stage of the life cycle or are induced to differentiate in culture, the basal body fails to duplicate and initiate cytokinesis, however, the nucleus continues to divide leading to large multinucleated cells that eventually die by a programmed cell death pathway [11]. The properties of the genetically defined centrin 1-deleted cell line $\left(\mathrm{LdCENl^{-1 }}\right)$ consisting of normal growth in the promastigote stage, but limited survival in the mammalian host due to intrinsic mechanisms, suggest possible utility as a live attenuated vaccine. The lack of virulence of this cell line and the ability to elicit a long-lasting protective, Th1 type response 
has been demonstrated in mice [13]; further supporting its development as a vaccine candidate.

A major concern with live attenuated parasite vaccines is the danger of reversion to a virulent parasite. Biomarkers are needed to assess the genetic and physiological traits of the organism. Further, these biomarkers must be measurable with an assay that is practical in a manufacturing setting.

Comprehensive genomic information about the attenuated parasite could be acquired with microarray technology. This genome-wide survey technique has been used effectively with Leishmania [14-15].

In this study, to identify gene expression patterns that could be biomarkers of attenuation, $L d C E N{ }^{--}$cells at an initial stage of differentiation to axenic amastigotes were compared to wild type cells at the same stage with a Leishmania donovani genomic microarray. Two genes, one coding for a $27 \mathrm{kDa}$ protein (p27) and another coding for putative Argininosuccinate Synthase (AS), were identified with a very clear pattern of expression that goes up with differentiation from the promastigote stage to the amastigote stage, but is sharply reduced in expression in the $L d C E N 1^{-/}$ amastigotes. The expression pattern for both the mRNA and protein levels of these genes was used as a measure of genetic stability in $L d C E N 1^{-/}$cells recovered from inoculated mice five weeks after injection and compared to the expression pattern of the original attenuated parasites.

\section{METHODS}

\subsection{Cell Culture}

Leishmania donovani Sudanese strain (Ld 1S) were cultured, alternating between the promastigote and axenic amastigote forms by changing the culture conditions as described [16]. L. donovani $\mathrm{LdCEN1}^{-/}$[11] were maintained in continuous culture as promastigotes. During differentiation experiments, for each replicate, $3.24 \times 10^{8}$ late log phase promastigotes $\left(1.74 \times 10^{7}\right.$ cells $\left./ \mathrm{ml}\right)$ were centrifuged, the promastigote culture medium removed and the cells resuspended in $32.4 \mathrm{ml}$ amastigote culture medium at $1 \times 10^{7} \mathrm{cells} / \mathrm{ml}$. The culture flask is kept in a $37^{\circ} \mathrm{C}$ incubator with $5 \% \mathrm{CO}_{2}$ to induce differentiation to axenic amastigotes. $L d C E N 1^{-/}$cells transfected with pXG-PHLEO-LdCEN were cultured as normal wild type cells in the presence of phleomycin (150 $\mu \mathrm{g} / \mathrm{ml})$ as described [11].

\subsection{RNA Preparation}

Total RNA was extracted from cultured Leishmania cells with RNA STAT-60 (TEL-TEST, Inc, Friendswood, TX) according to the manufacturer's instructions and further purified with RNeasy columns (Qiagen, Inc. Valencia, CA). RNA was used to synthesize fluorescent cDNA as described [17].

\subsection{Microarray Preparation, Hybridization and Analysis}

The microarray construction was previously described [14-15] and the array protocol can be found at http://www.ebi.ac.uk/microarray-as/aer/\#ae-main[0] by the accession number, A-MEXP-563. Feature intensities' $\log _{2}$ ratios were lowess normalized with the MIDAS software (http://www.tm4.org/midas.html), low intensity features filtered out and the remaining features were ranked by log ratio to select most differentially expressed clones as described [15]. The complete microarray data set is available in MIAME compliant format at ArrayExpress.org under accession \#E-MEXP-1825.

\subsection{Northern Blot Analysis}

RNA samples were fractionated as described [18] and hybridized with radioactively labeled inserts from the genomic library clones (46G8: Genbank/GSS/ED004307, 22C7: Genbank/GSS/CL844595). Northern blots were also hybridized with full length coding sequences for $\mathrm{Ld} \mathrm{p} 27$ and Ld Argininosuccinate Synthase (Ld AS). The quality and quantity of mRNA on the blots was assessed by hybridization with a Leishmania donovani alpha-tubulin cDNA probe [19]. Level of hybridization was quantified by exposing the blots to a PhosphorImager ${ }^{\circledR}$ (Molecular Dynamics).

\subsection{Antibody Production and Western Blotting}

The open reading frame sequences encoding $\mathrm{Ld} \mathrm{p} 27$ and Ld AS were amplified from $L$. donovani genomic DNA using primers designed from LinJ28_V3.1070 and LinJ23_V3.0300 (www.genedb.org) respectively (Table 1). The PCR products were cloned into pCRT7/CT-TOPO (Invitrogen Corporation, Carlsbad, CA), DNA sequence of the coding region confirmed, then protein expression performed in E. coli BL21 cells according to the plasmid manufacturer's instructions. Recombinant proteins were purified on Ni-NTA-agarose according to the manufacturer's protocol (Qiagen, Inc. Valencia, CA). Recombinant protein solutions were injected into New Zealand White rabbits according to the company protocol (Spring Valley Laboratories, Woodbine, MD). The antiserum raised against the p27 recombinant protein (anti-Ld p27) was analyzed by Western blot, which showed abundant reactivity to a $27 \mathrm{kDa}$ band in whole cell protein lysate of $L$. donovani cells. This antiserum was used at a dilution of 1:1000 in the experiments presented below. The antiserum raised against the Ld AS recombinant protein (anti-Ld AS) resulted in weak, but specific reactivity at approximately $46 \mathrm{kDa}$, the expected mass. The anti-Ld AS antiserum was further purified by affinity selection using recombinant $\mathrm{Ld} \mathrm{AS}$ bound to nitrocellulose membrane, eluted with $2.8 \mathrm{M} \mathrm{MgCl}_{2}$ and dialyzed into phosphate buffered saline resulting in higher reactivity. The affinity purified anti-AS was used at a 1:500 dilution for Western blots. Monoclonal anti-alpha-tubulin antibodies were obtained from Sigma (catalogue number T9026, Sigma, St. Louis, MO) and used at a 1:5,000 for Western blots.

Western blots of total cell lysates were performed as described [20] detecting the bound anti-p27 and anti-AS with anti-rabbit IgG conjugated to horseradish peroxidase at a dilution of $1: 10,000$.

\subsection{Cultured Macrophage Infection and Reverse Tran- scriptase, Real-Time PCR}

Elutriated monocytes obtained from normal human volunteers (under an approved research protocol for anonymous donations, National Institutes of Health Blood Bank) were differentiated in culture and infected as described [21]. Briefly, $1 \times 10^{6}$ macrophages were plated on a $50 \times 20 \mathrm{~mm}$ single chamber slide and $1 \times 10^{7}$ stationary phase promastigote parasites were exposed to the cells for 5 hours, then 
Table 1. Oligonucleotide Sequence

\begin{tabular}{|l|l|}
\hline Primer Name & Oligonucleotide Sequence (5'-3') \\
\hline \hline Subcloning & \\
p27 CDS forward & ATGTCTCGTTGCACGAACAAG \\
p27 CDS reverse & CACACCGTGGCCGGACAT \\
AS CDS forward & ATGCCTGCAACGGCGACGGAG \\
AS CDS reverse & CAAGCTGCTCGGCATCTCCTTCG \\
\hline$\underline{\text { Real-time PCR }}$ & \\
Ld p27 forward & CGGTCTCGGTGTTGGTGACG \\
Ld p27 reverse & CCAGATACGGCTCGTTCGC \\
Ld argininosuccinate synthase forward & GGAGGACCCAGCAAACGAGC \\
Ld argininosuccinate synthase reverse & GCGACTTCATCCCTACCAGGC \\
Ld alpha-tubulin forward & TTTTTTTTCGCCCTCGTCG \\
Ld alpha-tubulin reverse & GCCCCCCTCCACAGATACAC \\
human beta-actin forward & GATCATTGCTCCTCCTGAGC \\
human beta actin reverse & AAAGCCATGCCAATCTCATC \\
\hline
\end{tabular}

washed out with macrophage culture medium. A mock infection procedure involved innoculating the macrophage cell culture with a volume of promastigote culture supernatant equal to the volume of parasite suspension used for infection, but the mock infection medium was first centifuged at $10,000 \mathrm{~g}$ for $10 \mathrm{~min}$ to remove all live parasites. Forty hours post infection cells were washed with PBS and RNA STAT60 (Tel Test "B", Inc., Friendswood, TX) added directly to the culture plates, RNA was extracted according to the manufacturer's instructions. RNA was checked for integrity on a Bioanalyzer (Agilent Technologies, Waldbronn, Germany) and cDNA synthesized with an oligo dT primer and SuperScript II according to the manufacturer's instructions (Invitrogen, Inc., Carlsbad, CA). The cDNA was used as template in real time PCR performed in a SmartCycler (Cepheid, Sunnyvale, CA) with SYBR Green (Molecular Probes/Invitrogen, Carlsbad, CA) for detection of the PCR product and melt curve analysis to assure that only one fragment of the expected melting temperature was amplified. Primers are shown in Table 1. Three or more replicates of each determination were made. The cycle threshold $(\mathrm{Ct}$ value) for each gene in each RNA sample was recorded. A dilution series of known amounts of DNA with calculated gene copy number was used as template in real-time PCR reactions to construct a standard curve that enabled conversion of $\mathrm{Ct}$ values to copy numbers. The copy number values were adjusted by dividing the gene-specific copy number by the Ld tubulin copy number to normalize for the number of parasites in the infected sample and dividing by the human beta-actin copy number to normalize for the amount of cDNA synthesized in the reverse transcriptase reaction. Thus the adjusted copy number value should represent the relative amount of p27 or AS expressed per parasite in each sample.

\subsection{Animal Infection Studies}

All procedures performed in live animals were done according to an approved protocol under the review of the Center for Biologic Evaluation and Research Institutional Animal Care and Use Committee.
The preparation of infective metacyclics from $\mathrm{LdCEN1}^{-1}$ cultures was performed as described previously [22] with the following modifications. Stationary phase promastigotes were separated using a step gradient. Approximately 1 X $10^{8}$ cells in culture medium were layered on 10\% Ficoll in M199 medium, which overlays $20 \%$ Ficoll. After centrifugation at $1350 \mathrm{~g}$ for 15 minutes at $4^{\circ} \mathrm{C}$, the liquid from the top down to most of the $10 \%$ ficoll layer, avoiding the thick layer of material on top of $20 \%$ layer, was collected and the cells pelleted and washed once with M199 medium and re-suspended in $250 \mu \mathrm{l}$ of the same medium. Metacyclic stage cells can be distinguished from procyclic stage by their more rapid movement, smaller body size and longer flagellum. Cells fitting this description were counted microscopically using a hemocytometer. Three million $L d C E N 1^{-/-}$metacyclics were injected into 5-7 week old BALB/c mice via the tail vein. Five weeks after injection, spleens from mice were isolated, homogenized and parasite burden measured by limiting dilution [23]. Serial dilution of spleen cell suspensions resulted in outgrowth of promastigote cells from the mice infected with $\mathrm{LdCEN1}^{-/}$parasites, as described elsewhere [13]. The promastigotes recovered from the mice infected with $L d C E N 1^{-1}$ parasites were expanded in culture to a total of $3 \times 10^{8}$ cells per replicate, a sufficient quantity for biochemical studies, then shifted to amastigote culture conditions to induce differentiation into axenic amastigotes in parallel with $L d C E N 1^{-/}$cells kept continuously in culture and wild type cells. RNA and protein samples from these parasites were used in our experiments to confirm the expression pattern of the biomarkers. Such RNA and protein samples were prepared four independent times to provide sufficient replicates for statistical comparison.

\section{RESULTS}

\subsection{Identification of Potential Biomarkers}

To identify genes other than centrin that are altered in their expression after differentiation of the $\mathrm{LdCEN1}^{-/}$cell line, total RNA samples were collected from these parasites 
40 hours after shifting from promastigote to the amastigote culture conditions. RNA was also collected from wild type parasites 40 hours after shifting to the amastigote culture (see methods). The wild type parasites were placed in culture at saturation cell density to match as nearly as possible the stalled growth that occurs in the $L d C E N 1^{-/}$cell line.

To assess changes in gene expression, $\mathrm{LdCEN1}^{-/}$axenic amastigote RNA was compared to wild type stationary axenic amastigote RNA by two color fluorescence hybridization to a microarray. The array has 4224 genomic clones printed in triplicate with known gene and negative controls [14-15]. This subset of the genome can be estimated to hybridize with about $37 \%$ of the expressed genes [24]. The mean ratio of $L d C E N 1^{--}$fluorescence intensity to wild type intensity was used to rank each clone on each of the four hybridizations performed. The top 10-14 ranking over expressed and top 10-14 ranking under expressed clones were identified that had at least two features with rankings in the top 100 features.

The top ranking differentially expressed clones from the 4 hybridizations were compared and 30 clones were selected that ranked in more than one hybridization. The Leishmania DNA insert of each of these clones was sequenced and searched against the $L$. infantum genome (www.genedb.org/genedb/leish/blast.jsp). All of the clones could be unambiguously assigned to a position in the $L$. infantum genome due to the high level of conservation between these two species that cause visceral disease. Thirteen of the clones, all showing higher expression in the $L d C E N I^{-/-}$ axenic amastigotes, represent telomeric and subtelomeric fragments of ten different chromosomes. Telomeric clones have been identified as differentially expressed in other Leishmania genomic microarray studies [25-26]. Of the remaining differentially expressed clones selected, one is part of a pseudogene, seven align in or very near the coding sequence of hypothetical proteins and nine align in or near a coding sequence that has homology to a known gene. The telomere clones were eliminated from further consideration because the transcription of telomeric sequence is of uncertain significance and Northern blots with repetitive probes are nonspecific. Some of the other clones were eliminated because the sequence indicated a position too remote from a coding sequence to be certain of the expressed gene or an encoded protein that would be too large to be suitable for a practical biomarker assay. Northern blot analysis (data not shown) revealed some of the clones were not differentially expressed, so they were eliminated from consideration.

\section{2. mRNA Expression as a Biomarker}

Two clones were selected that had potential to be biomarkers of attenuation. One of the clones, 46G8, aligns $1,872 \mathrm{bp}$ on the 3 ' side of an open reading frame annotated as LinJ28_V3.1070 in the L. infantum genome and also was identified in a microarray comparison of amastigote and promastigote RNA [14]. This coding sequence was cloned from the $L$. donovani genome by PCR using primers designed from the $L$. infantum sequence (Table 1). Due to the lack of homology with any known gene, this coding sequence will be designated the p27 gene in recognition of the $27 \mathrm{kDa}$ putative protein that it would encode. Northern blot analysis with the coding sequence probe shows that the amastigote stage expresses a substantially higher level of this mRNA than the promastigote stage and the $L d C E N 1^{-/}$amastigotes express a very low level (Fig. 1A, top row, lanes 1-3). RNA loading was measured by hybridization of the same blot with Ld alpha-tubulin and was seen to vary somewhat (Fig. 1A, lower row). To evaluate the relative level of expression, the PhosporImager volume of each band was normalized to tubulin and the normalized level of the mean of at least 3 different RNA hybridizations displayed in the bar graph in Fig. (1B).

To evaluate whether the potential biomarker expression remains stable during infection of the host, $L d C E N 1^{-/-}$parasites were recovered from mice 5 weeks after infection. Recovered promastigotes were differentiated into axenic amastigotes in vitro and RNA samples evaluated on Northern blots. These recovered $L d C E N I^{-/}$parasites have retained the characteristic of expressing a low level of p27 mRNA when they differentiate into axenic amastigotes (Fig. 1A, lane 4 and Fig. 1B). To demonstrate that the low expression level of p27 is a specific indicator of the absence of centrin, the centrin protein was expressed from an episome transfected into the $L d C E N 1^{-1-}$ cells. Axenic amastigotes of this "add back" cell line have restored a near wild type level of p27 mRNA expression (Fig. 1A, lane 5 and Fig. 1B).

The second clone, $22 \mathrm{C} 7$, aligns with the 3 ' end of the coding sequence and the 3'UTR of LinJ23_V3.0300 in the $L$. infantum genome database. This annotated open reading frame encodes a protein that is $59.7 \%$ similar to the human Argininosuccinate Synthase (AS), an enzyme in the mammalian urea cycle. The full length coding sequence was amplified from L. donovani genomic DNA, cloned and used as a probe on Northern blots. The expression pattern revealed the axenic amastigote stage expresses a substantially higher level of this mRNA than the promastigote stage and the $L d C E N 1^{-1-}$ amastigotes express a very low level (Fig. 2A, top row, lanes 1-3). The differences were not due to RNA loading as indicated by the tubulin hybridization of the same blots (Fig. 2A, bottom row), but mRNA loading varied somewhat. The relative level was calculated as for p27 and the mean of at least 3 RNA hybridizations shown in the bar graph (Fig. 2B). Similar to p27, $\mathrm{LdCENI}^{-/}$parasites that were recovered from infected mice continue to express a low level of AS mRNA when they differentiate into axenic amastigotes (Fig. 2A, lane 4 and Fig. 2B) and the centrin transfected $L d C E N 1^{--}$cells (CenKO AB) express a near wild type level of AS mRNA (Fig. 2A, lane 5 and Fig. 2B). Thus measuring the mRNA levels of both p27 and AS genes could serve as biomarkers of attenuation for $L d C E N 1^{-/}$cells.

\subsection{Protein Expression as a Biomarker}

Next, we measured the protein levels of p27 and Ld AS in $L d C E N 1^{-/}$parasites using antibodies specific to these proteins (see Methods). Preimmune sera did not react with any L. donovani proteins (data not shown). The anti-Ld p27 stains a band approximately $27 \mathrm{kDa}$ in size that is expressed at a substantially higher level in axenic amastigotes than in promastigotes (Fig. 3A, Lanes 1 and 2, top row). The differences in expression are not due to loading of the protein samples as indicated by binding the same blot with antitubulin antibody (Fig. 3A, bottom row). Surprisingly, unlike the mRNA, the protein level is not reduced in $L d C E N 1^{-1-}$ 


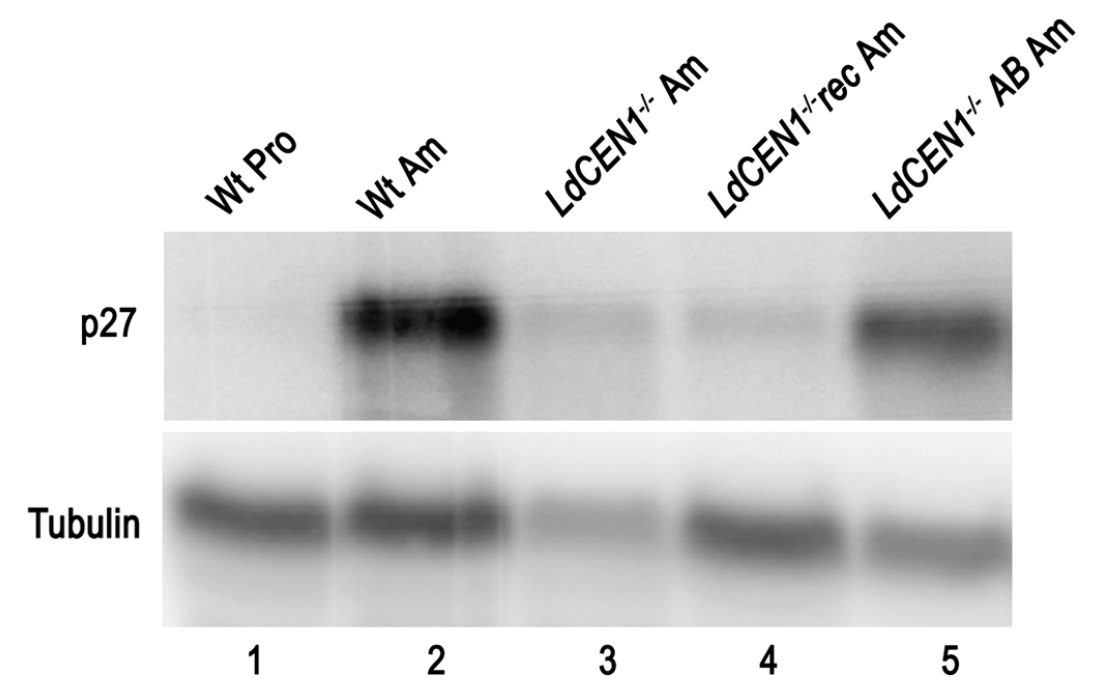

(a)

Mean Relative p27 Expression

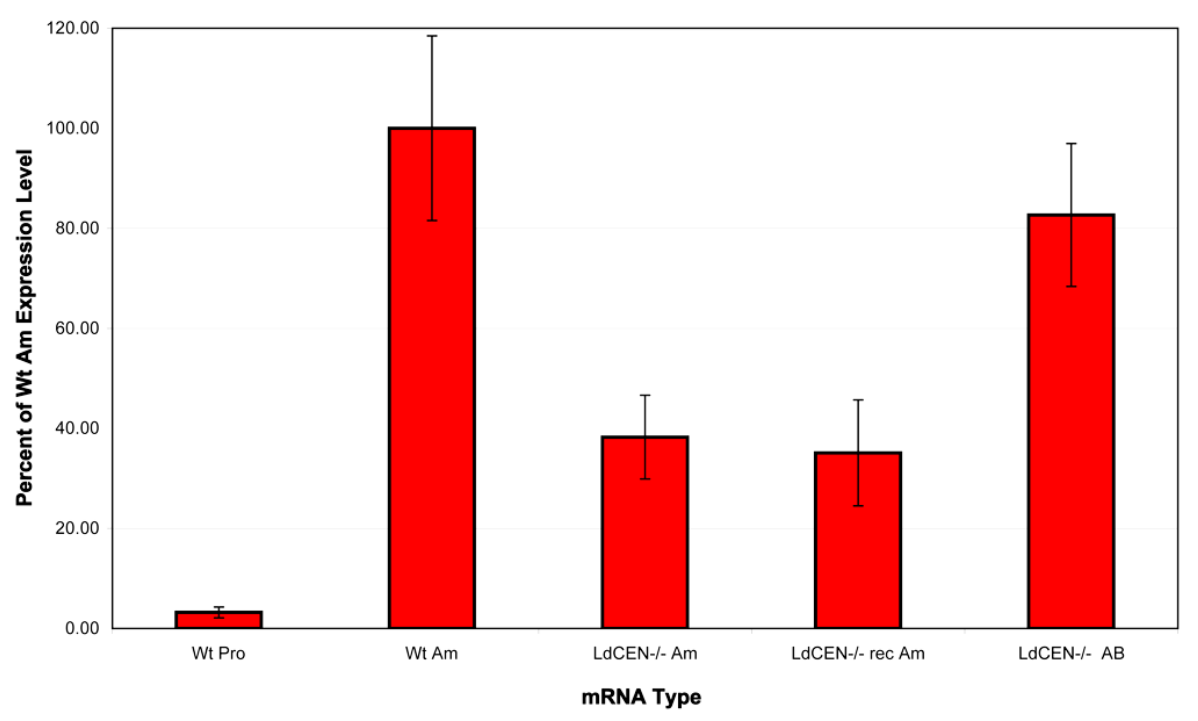

(b)

Fig. (1). mRNA expression of p 27 correlates with attenuation. Northern blots of total RNA extracted from the indicated Leishmania donovani cell types. Hybridization was performed with the coding sequence probe for the Ld p27 gene revealing a band of approximately $3.3 \mathrm{~kb}$, stripped and rehybridized with a probe for Ld alpha-tubulin. (A) Image of a representative blot showing p27 hybridization in the upper panel and tubulin hybridization in the lower panel. (B) Bar graph showing mean normalized p27 expression level quantified by PhosphorImager. Values for each cell type are displayed as the percent of the mean wild type amastigote level. Each bar is the mean of at least three independent RNA preparations with error bars showing +/- the standard error of the mean. Lane 1: Wt Pro=wild type promastigotes, Lane 2: Wt $\mathrm{Am}=$ wild type axenic amastigotes, Lane 3: $L d C E N 1^{-/} \mathrm{Am}=$ centrin1-deleted axenic amastigotes, Lane 4: $L d C E N 1^{-/}$rec $=$centrin 1 -deleted axenic amastigotes derived from parasites recovered from mice, Lane 5: LdCEN1 ${ }^{-/} \mathrm{AB} A \mathrm{Am}=$ centrin1-deleted axenic amastigotes carrying an episomal centrin gene.

amastigotes (Fig. 3A, Lane 3, top row). Suspecting that the decrease in protein occurred later than the RNA, protein lysates were collected from wild type and $\mathrm{LdCEN1}^{-/-}$parasites at a later time after shifting promastigotes to the axenic amastigote culture conditions. At 94 hours, a time when the majority of $L d C E N 1^{-/-}$cells are multi-nucleated [11], the p27 protein is still as abundant as wild type (data not shown). The p27 protein level was also measured in $L d C E N 1^{-/-}$re- covered from mice differentiated into axenic amastigotes (Fig. 3A, Lane 4, top row) and cells that were transfected with a centrin expression plasmid (Fig. 3, lane 5, top row). The $\mathrm{p} 27$ protein expression in these cells was similar to wild type as expected.

Western blot analysis with anti-Ld AS revealed a band at approximately $46 \mathrm{kDa}$, the expected mass of the predicted polypeptide (Fig. 3B, Lane 2, top row). The level of expres- 


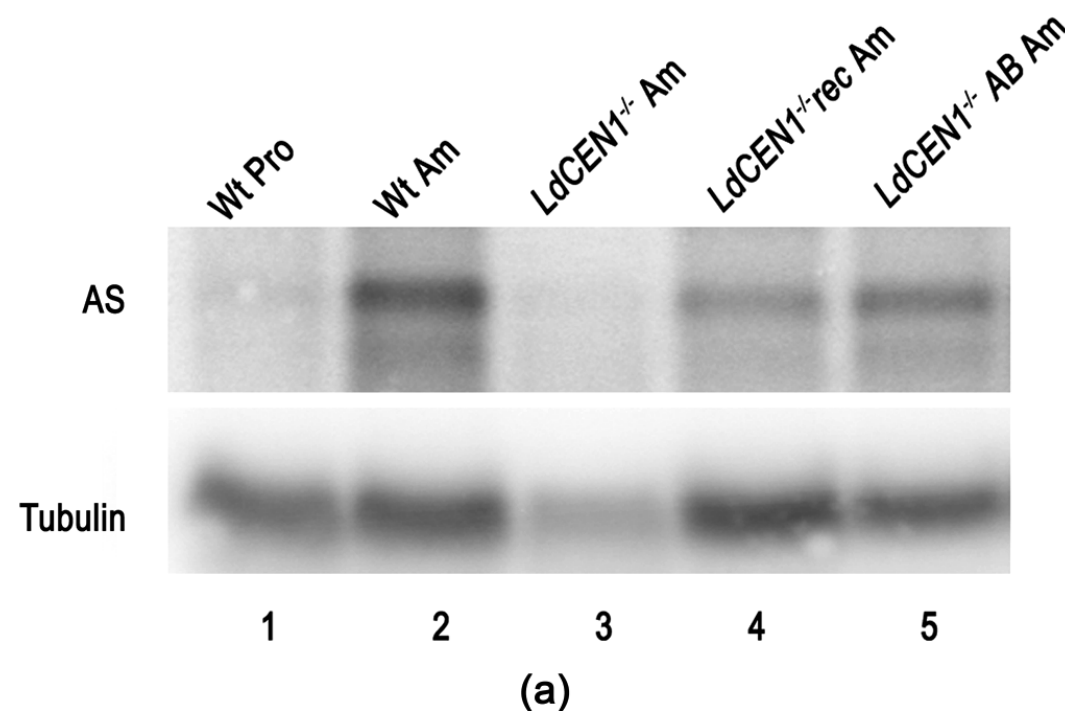

Mean Relative AS Expression

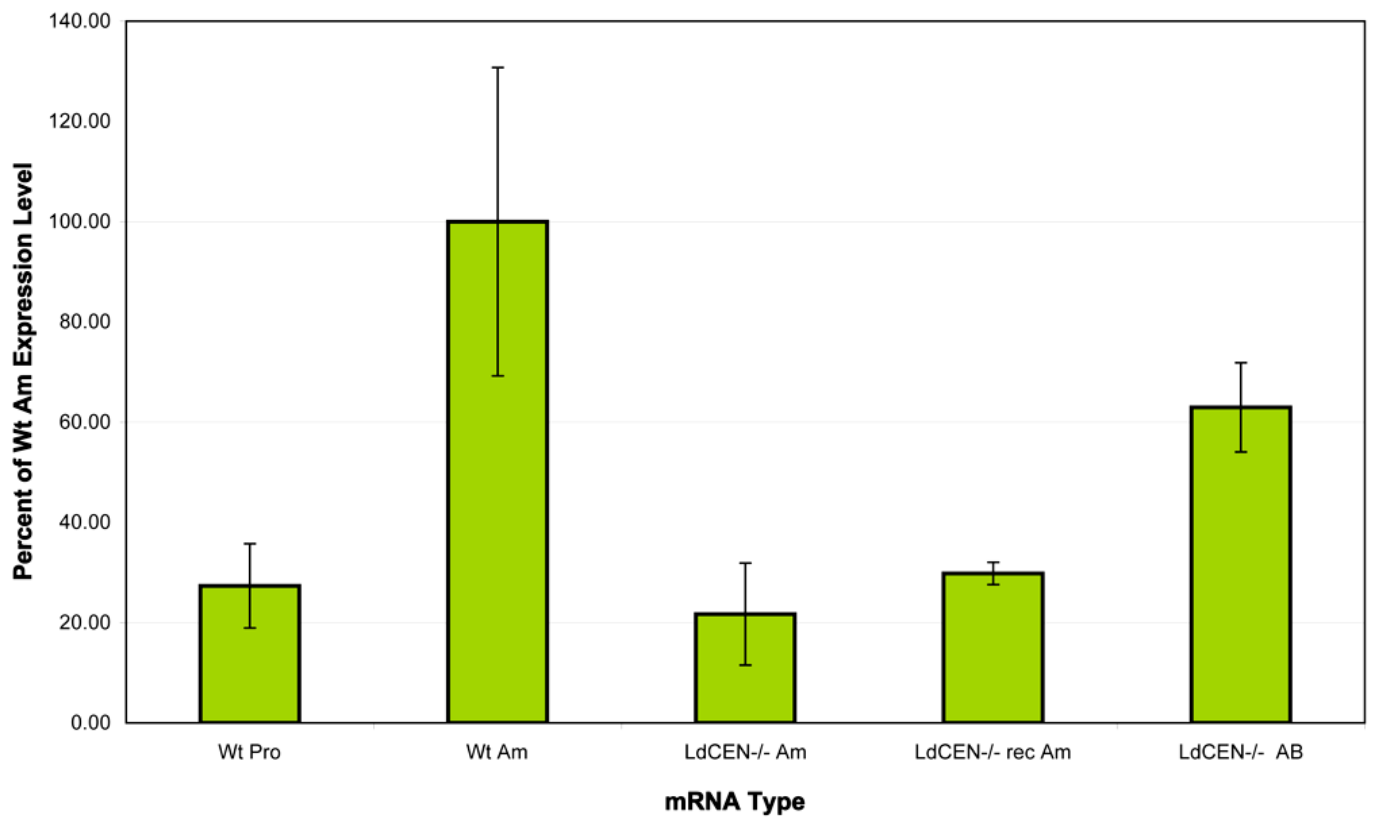

(b)

Fig. (2). mRNA expression of Ld argininosuccinate synthase correlates with attenuation. Northern blots of total RNA extracted from the indicated Leishmania donovani cell types. Hybridization was performed with the the coding sequence probe for the Ld putative Argininosuccinate Synthase coding sequence revealing a band of 3.7kb, stripped and rehybridized with a probe for Ld alpha-tubulin. (A) Image of a representative blot showing AS hybridization in the upper panel and tubulin hybridization in the lower panel. (B) Bar graph showing mean normalized AS expression level quantified by PhosphorImager. Values for each cell type displayed as the percent of the mean wild type amastigote level. Each bar is the mean of at least three independent RNA preparations for each sample type with error bars showing $+/-$ the standard error of the mean. Lane 1: Wt Pro=wild type promastigotes, Lane 2: Wt Am=wild type axenic amastigotes, Lane 3: LdCEN1 ${ }^{-/}$Am=centrin1deleted axenic amastigotes, Lane 4: $L d C E N 1^{-/}$rec=centrin 1-deleted axenic amastigotes derived from parasites recovered from mice, Lane 5: $L d C E N 1^{-/} \mathrm{AB} \mathrm{Am}=$ centrin1-deleted axenic amastigotes carrying an episomal centrin gene.

sion was consistent with the mRNA levels: low in promastigotes, high in amastigotes, but low in $\mathrm{LdCENI}^{-/}$amastigotes (Fig. 3B, Lanes 1, 2 and 3, top row). The $L d C E N 1^{-/}$ parasites recovered from infected mice retained the charac- teristic of low AS protein expression when they were differentiated to axenic amastigotes (Fig. 3B, lane 4, top row). Further, the expression of AS protein was restored by episomal centrin in the $L d C E N 1^{-/}$parasites indicating the 


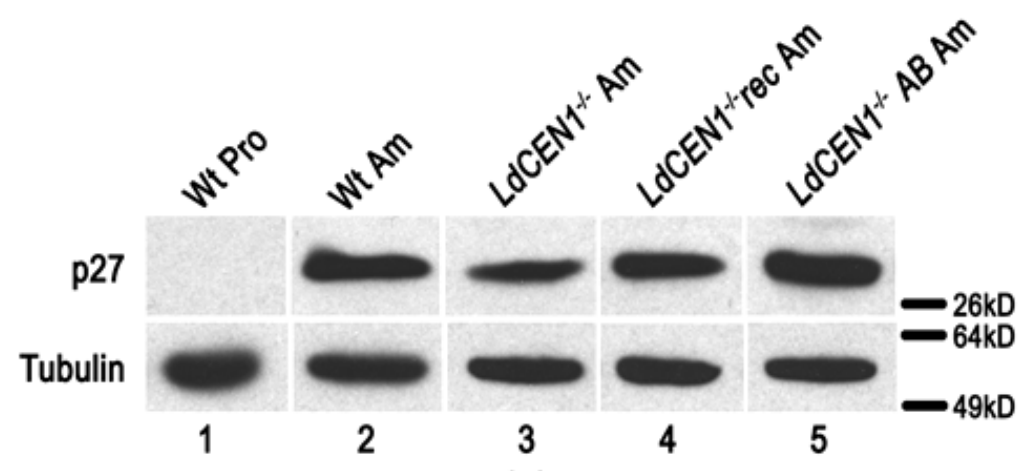

(a)

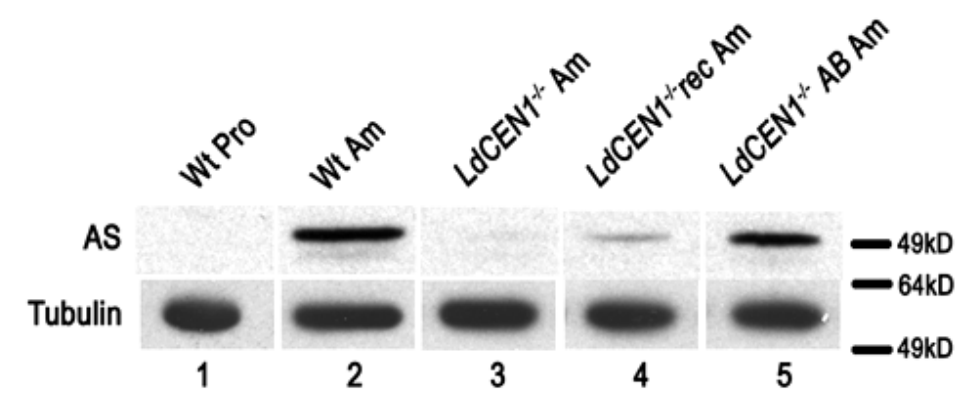

(b)

Fig. (3). Protein expression as a biomarker of attenuation. Western blots of total protein lysates of L. donovani cells of the cell types indicated. (A) Blot was bound with antiserum to Ld p27 (upper row), then stripped and rebound with monoclonal antibody to chicken alphatubulin (lower row). (B) Blot was bound with affinity purified antibodies to Ld AS (upper row), stripped and rebound with monoclonal antibody to chicken alpha-tubulin (bottom row). Images are representative of at least three independent protein samples for each sample type. Lane 1: wild type promastigotes, Lane 2: wild type axenic amastigotes, Lanes 3: centrin1-deleted axenic amastigotes, Lane 4: centrin1deleted axenic amastigotes derived from parasites recovered from mice, Lane 5: centrin1-deleted axenic amastigotes carrying an episomal centrin gene. Five micrograms of total protein were loaded in each lane.

low protein expression is also a specific result of the absence of centrin. Thus the AS, but not the p27 protein level could serve as a biomarker of attenuation.

\subsection{Biomarker Expression Level in Infected Human Macrophages}

Though biomarker expression levels were evaluated in parasites recovered from mice, it is important to recognize that these were recovered as promastigotes, differentiated to amastigotes in culture as the axenic form and samples extracted from pure parasites. To evaluate whether biomarker expression levels seen in the cultured parasites were representative of expression in intracellular amastigotes and could be measured in a tissue sample, the various Leishmania cell types were used to infect elutriated human monocytes that were differentiated into macrophages during a week of culture with Macrophage Colony Stimulating Factor. Forty hours after infection, cells were harvested and RNA extracted. A reverse transcriptase reaction converted RNA to cDNA. The cDNAs were used as templates in real time PCR reactions using gene-specific primer pairs (Table 1) and SYBR Green intercalating dye for detection of the PCR product. To evaluate biomarker expression, RNA samples from uninfected macrophages, macrophages infected with wild type, continuously cultured $L d C E N 1^{-/}, L d C E N 1^{-/-}$recovered from mice and $L d C E N 1^{-/}$episomally expressing centrin were subject to PCR with Ld p27 primers, Ld AS primers,
Ld tubulin primers and human beta-Actin primers. Ld tubulin served to normalize for parasite load and the human betaActin normalized for efficiency of RNA extraction and cDNA synthesis. The p27 primers showed no measurable background in the mock infected macrophages and the pattern of expression levels resembled that seen in the cultured amastigotes (Fig. 4A). Similarly, the AS primers showed no background in the mock infected cells and a pattern of expression levels similar to cultured amastigotes (Fig. 4B). Thus, both p27 and AS could be used as biomarkers of attenuation in infected tissue samples.

\section{DISCUSSION}

The $\mathrm{LdCEN1}^{-/-}$cell line of Leishmania donovani has potential as a vaccine candidate because it can be propagated in culture in the promastigote stage, but when it differentiates to the amastigote stage in the mammalian macrophage, it fails to divide and after surviving for a short period of time, dies by an intrinsic mechanism [11]. The demonstrated ability of the $\mathrm{LdCEN1}^{-/-}$cell line to confer long lasting protection in mice [13] suggests continued development as a vaccine candidate. As a live attenuated parasite vaccine, this cell line must be monitored for genetic stability as it is carried in culture, expanded to production levels and used as a vaccine to inoculate recipients. In addition to monitoring the obvious presence or absence of $L d C E N 1$, other genes that are uniquely altered in expression in the $L d C E N 1^{--}$amastigotes 
Intracellular p27 Expression hActB and Ld tubulin Normalized

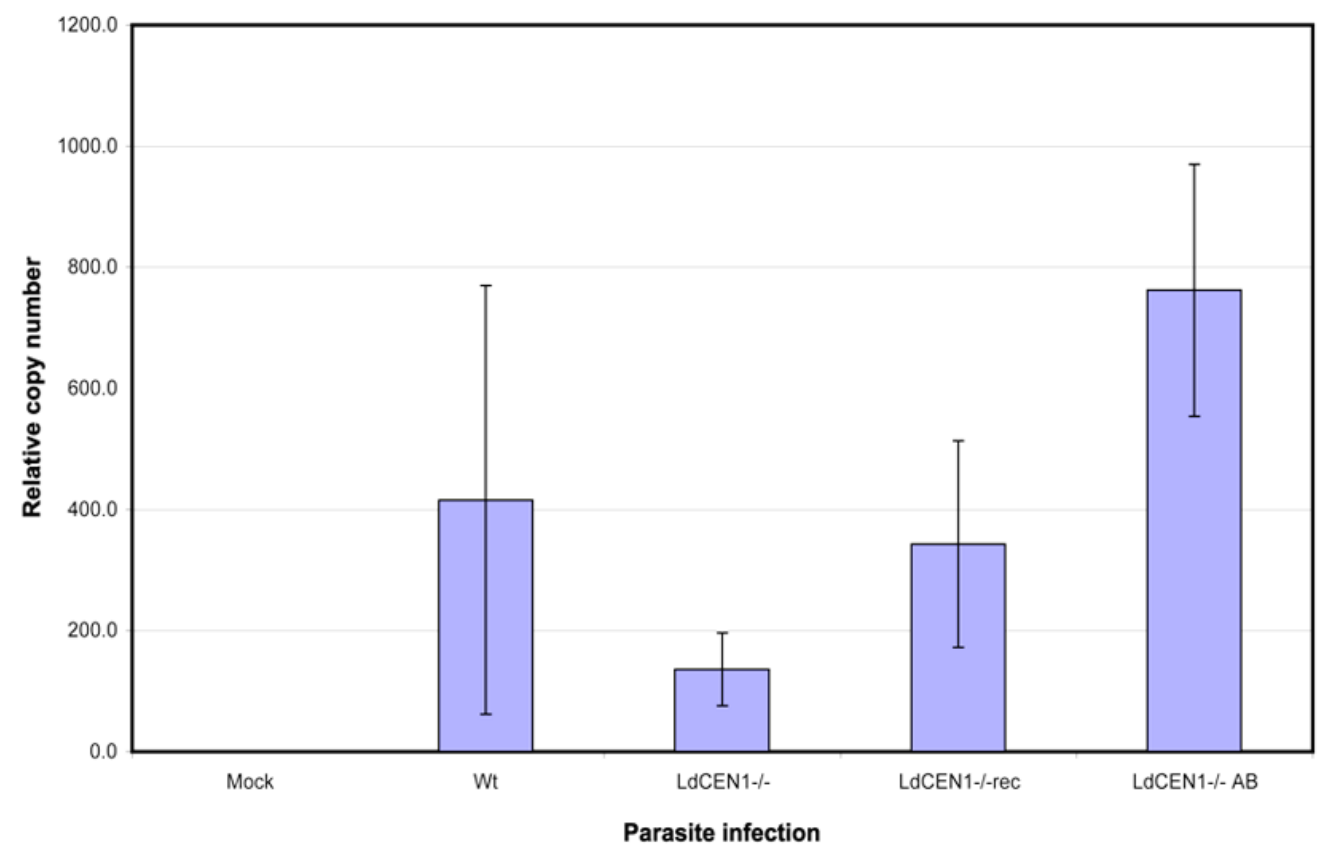

(a)

Intracellular AS Expression hActB and Ld tubulin Normalized

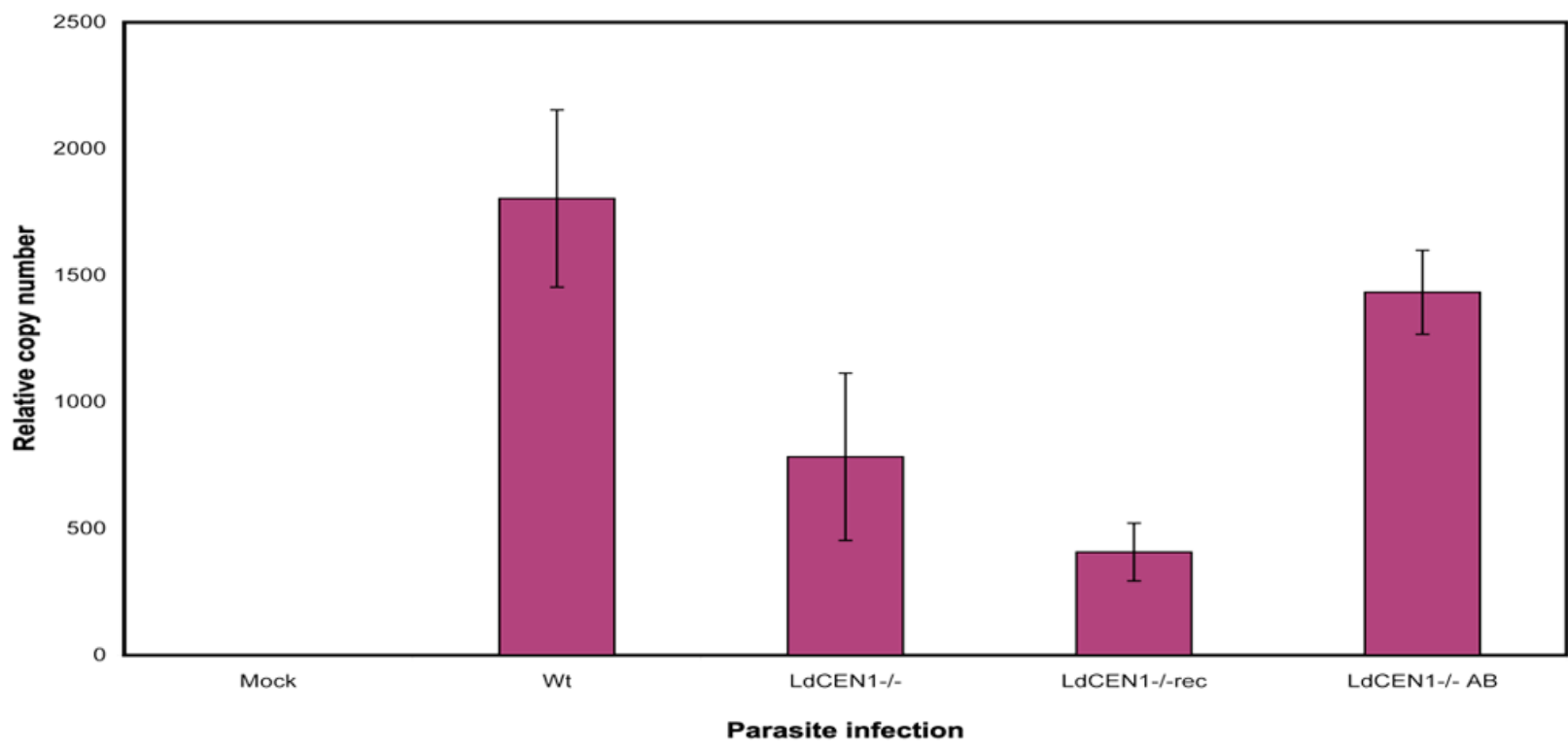

(b)

Fig. (4). Intracellular expression level of biomarkers. Bar graphs indicating the copy number of each biomarker measured by reverse transcriptase real-time PCR in total RNA samples from infected macrophages. (A) Amplification with primers specific for the p27 gene. (B) Amplification with primers for the Ld AS gene. Column labels indicate result with RNA sample from macrophages infected with: Mockuninfected, Wt-wild type parasites, $L d C E N 1^{-/}$-centrin gene deleted parasites, $L d C E N 1^{-/}$rec-centrin gene deleted parasites recovered from mice, $L d C E N 1^{-/} \mathrm{AB}$ - centrin gene deleted parasites transfected with a centrin expressing plasmid. 
provide biomarkers of attenuation. The $L$. donovani $\mathrm{p} 27$ gene and the putative Argininosuccinate Synthase gene have been demonstrated to have the properties that allow them to serve as such biomarkers.

The level of p27 mRNA expression could be easily measured after in vitro differentiation to axenic amastigotes by reverse transcriptase-PCR (RT- PCR) to indicate that the centrin deleted cells have retained the attenuated phenotype at various stages of vaccine development. More importantly, examination of RNA prepared from bone marrow aspirates of kala-azar patients with RT-PCR using primers specific for the Ld p27 gene revealed a specific band not seen in uninfected individuals (Sharma, et al., manuscript in preparation $^{1}$ ) indicating the presence of the p27 transcript in the infected samples. Thus similar samples could be taken from vaccinees receiving the $L d C E N 1^{-/}$parasites to show that the p27 gene has not returned to wild type expression levels. However, the protein expression level for $\mathrm{p} 27$ remains at the wild type level in the $L d C E N 1^{-/}$parasites even though the mRNA is substantially reduced, making the protein ineffective as a biomarker of attenuation. The discrepancy between the p27 mRNA and protein level at 40 or more hours after shifting promastigotes to the amastigote culture conditions can be explained by very early expression of p 27 protein soon after differentiation to the amastigote form, before the failure of cell division (p27 protein has been detected within 3 hours of infection of mouse peritoneal macrophages, Dey et al., manuscript in preparation ${ }^{2}$ ). Furthermore, once p27 protein has been synthesized, post transcriptional and post translational mechanisms may stabilize this protein so that the protein level and the mRNA level are no longer correlated as has been described in Leishmania [27] and other organisms [28].

The level of Ld AS mRNA could also be readily measured in cultured $L d C E N l^{-/}$cells after differentiation to axenic amastigotes to indicate that the cell line had not lost its attenuated phenotype, as demonstrated by the RT-PCR with infected macrophages in this study. RT-PCR for Ld AS in human leishmaniasis patients has not been tested, but the successful amplification of Ld AS in the infected human macrophage samples indicates that the human genomic DNA does not cross hybridize or interfere. Thus, measurement of Ld AS expression in the tissues of vaccinees could be possible. The loss of expression of the AS protein and the specific antibody for that protein suggest that high throughput assays such as antigen capture ELISA could be designed for screening $L d C E N 1^{-1 /}$ amastigote cells at various points in the development of a live attenuated vaccine as another biomarker of attenuation.

Other genetically altered Leishmania cell lines have been evaluated for reduced virulence that would indicate potential as live attenuated vaccines and several have been tested in model species and showed protection from challenge with virulent parasites (reviewed in [29]) though only one has

${ }^{1}$ Differential expression of Leishmania genes contribute to tissue tropism and pathogenicity in leishmaniasis. Paresh Sharma, Srividya Gurumurthy, Robert Duncan, Hira L.Nakhasi and Poonam Salotra. Institute of Pathology (ICMR), Safdarjung Hospital Campus, New Delhi, India-110029.

${ }^{2}$ Characterization of Leishmania donovani amastigote specific protein and its role in virulence. Ranadhir Dey, Hira Nakhasi, Robert Duncan. FDA/CBER, Bethesda, MD 20892 been taken forward to primate testing [30] where it did not show protection. To the present, none of these genetically targeted attenuated Leishmania cell lines has been evaluated for genetic stability. Significantly, follow up of $\operatorname{lpg} 2^{-} \mathrm{mu}-$ tants, which lacked virulence, showed that after 125 days of infection in mice, these parasites were partially reverted to virulence even in the absence of LPG2-dependent glycoconjugates [31]. Some genetically targeted live attenuated viral vaccines have been developed with specific alterations of base sequence in the genome. These have been monitored for genetic stability by sequencing the altered site in the recovered vaccine organism [32] or RT-PCR and restriction analysis [33]. Yet even these are only directed at the targeted mutations themselves and would not detect compensatory changes at other loci. Our study is unique in having identified genes from sites remote from the targeted deletion that can monitor more broadly the genetic stability of the $L d C E N I^{-/}$ live attenuated parasite vaccine candidate. Such biomarkers must be developed because they could be indicators of compensatory mutations arising in the genome at a distance from the targeted alteration. Additional biomarkers for the $L d C E N I^{-/}$strain could also be identified by using a microarray with more extensive genome coverage. Differentially expressed genes may remain in the genome that were not identified by the DNA fragments on the array used in this study.

Immunological studies have led to the conclusion that achieving protection from infection will require Leishmania parasites to survive in the host for a period of time [29]. The recovery of live $L d C E N 1^{-/}$cells after 5 weeks of infection in mice indicates this attenuated parasite may be effective. However, the ability of the Leishmania parasite to adapt under the selective pressure to survive in the human host is demonstrated by the number of drug resistant strains that have arisen [1]. Gene deletion mutants like $L d C E N 1^{-/}$, which dies after a short period in the host by an intrinsic mechanism (failure to divide leading to programmed cell death [11], have the best potential to exhibit the safety profile demanded for clinical use. However, monitoring biomarkers of attenuation such as those described here as well as further genetic evaluation to identify any compensatory mutations that could arise will be an important aspect of any future development as a live attenuated Leishmania vaccine.

\section{ABREVIATIONS}

\begin{tabular}{|c|c|c|}
\hline AS & $=$ & argininosuccinate synthase \\
\hline cDNA & $=$ & copy DNA \\
\hline $\mathrm{Ct}$ & $=$ & cycle threshold \\
\hline ELISA & $=$ & enzyme linked immunosorbent assay \\
\hline $\mathrm{kDa}$ & $=$ & kilodalton \\
\hline$L d C E N 1^{--}$ & $=$ & $\begin{array}{l}\text { Leshmania donovani centrin gene deleted } \\
\text { cell line }\end{array}$ \\
\hline LPG2 & $=$ & Golgi GDP-mannose transporter \\
\hline RT-PCR & - & $\begin{array}{l}\text { reverse transcriptase polymerase chain reac- } \\
\text { tion }\end{array}$ \\
\hline Th1 & & $\mathrm{T}$ helper one \\
\hline
\end{tabular}




\section{ACKNOWLEDGEMENTS}

We express our appreciation for critical review of the manuscript to Alain Debrabant and Sanjai Kumar. We thank Austin Fang, Neha Kumar and Kyrstin Fornace for technical assistance.

\section{REFERENCES}

[1] Murray HW, Berman JD, Davies CR, Saravia NG. Advances in leishmaniasis. Lancet 2005; 366(9496): 1561-77.

[2] Khalil EA, El Hassan AM, Zijlstra EE, et al. Autoclaved Leishmania major vaccine for prevention of visceral leishmaniasis: a randomised, double-blind, BCG-controlled trial in Sudan. Lancet 2000; 356(9241): 1565-9.

[3] Connell ND, Medina-Acosta E, McMaster WR, Bloom BR, Russell DG. Effective immunization against cutaneous leishmaniasis with recombinant bacille Calmette-Guerin expressing the Leishmania surface proteinase gp63. Proc Natl Acad Sci USA 1993; 90(24): 11473-7.

[4] Skeiky YA, Coler RN, Brannon M, et al. Protective efficacy of a tandemly linked, multi-subunit recombinant leishmanial vaccine (Leish-111f) formulated in MPL adjuvant. Vaccine 2002; 20(2728): 3292-303.

[5] Walker PS, Scharton-Kersten T, Rowton ED, et al. Genetic immunization with glycoprotein $63 \mathrm{cDNA}$ results in a helper T cell type 1 immune response and protection in a murine model of leishmaniasis. Hum Gene Ther 1998; 9(13): 1899-907.

[6] Iborra S, Soto M, Carrion J, Alonso C, Requena JM. Vaccination with a plasmid DNA cocktail encoding the nucleosomal histones of Leishmania confers protection against murine cutaneous leishmaniasis. Vaccine 2004; 22(29-30): 3865-76.

[7] Ghosh A, Labrecque S, Matlashewski G. Protection against Leishmania donovani infection by DNA vaccination: increased DNA vaccination efficiency through inhibiting the cellular p53 response. Vaccine 2001; 19(23-24): 3169-78.

[8] Kedzierski L, Zhu Y, Handman E. Leishmania vaccines: progress and problems. Parasitology 2006; 133 Suppl: S87-112.

[9] Palatnik-de-Sousa CB. Vaccines for leishmaniasis in the fore coming 25 years. Vaccine 2008; 26(14): 1709-24.

[10] Silvestre R, Cordeiro-da-Silva A, Ouaissi A. Live attenuated Leishmania vaccines: a potential strategic alternative. Arch Immunol Ther Exp (Warsz) 2008; 56(2): 123-6.

[11] Selvapandiyan A, Debrabant A, Duncan R, et al. Centrin gene disruption impairs stage-specific basal body duplication and cell cycle progression in Leishmania. J Biol Chem 2004; 279(24): 25703-10.

[12] Wiech H, Geier BM, Paschke T, et al. Characterization of green alga, yeast, and human centrins. Specific subdomain features determine functional diversity. J Biol Chem 1996; 271(37): 2245361.

[13] Selvapandiyan A, Dey R, Nylen S, Duncan R, Sacks D, Nakhasi HL. Intracellular replication-deficient Leishmania donovani induces long lasting protective immunity against visceral leishmaniasis. J Immunol 2009; 183(3): 1813-20.

[14] Srividya G, Duncan R, Sharma P, Raju BV, Nakhasi HL, Salotra P. Transcriptome analysis during the process of in vitro differentiation of Leishmania donovani using genomic microarrays. Parasitology 2007; 134(Pt 11): 1527-39.

[15] Duncan R, Salotra P, Goyal N, Akopyants N, Beverley S, Nakhasi $\mathrm{H}$. The application of gene expression microarray technology to kinetoplastid pathogenesis. Curr Mol Med 2004; 4: 611-21.

[16] Debrabant A, Joshi MB, Pimenta PFP, Dwyer DM. Generation of Leishmania donovani axenic amastigotes: their growth and biological characteristics. Int J Parasitol 2004; 34(2): 205-17.
[17] Han J, Lee H, Nguyen NY, Beaucage SL, Puri RK. Novel multiple 5'-amino-modified primer for DNA microarrays. Genomics 2005; 86(2): 252-8

[18] Duncan R, Alvarez R, Jaffe C, et al. Early response gene expression during differentiation of cultured Leishmania donovani. Parasit Res 2001; 87: 897-906

[19] Joshi M, Dwyer DM, Nakhasi HL. Molecular cloning and characterization of a Leishmania donovani alpha-tubulin gene. J Eukaryot Microbiol 1995; 42(5): 628-32.

[20] Duncan R, Collins I, Tomonaga T, Zheng T, Levens D. A unique transactivation sequence motif is found in the carboxyl-terminal domain of the single strand binding protein FBP. Mol Cell Biol 1996; 16(5): 2274-82.

[21] Gannavaram S, Vedvyas C, Debrabant A. Conservation of the proapoptotic nuclease activity of endonuclease $\mathrm{G}$ in unicellular trypanosomatid parasites. J Cell Sci 2008; 121(Pt 1): 99-109.

[22] Spath GF, Beverley SM. A lipophosphoglycan-independent method for isolation of infective Leishmania metacyclic promastigotes by density gradient centrifugation. Exp Parasitol 2001; 99(2): 97-103.

[23] Titus RG, Marchand M, Boon T, Louis JA. A limiting dilution assay for quantifying Leishmania major in tissues of infected mice. Parasite Immunol 1985; 7(5): 545-55.

[24] Akopyants NS, Clifton SW, Martin J, et al. A survey of the Leishmania major Friedlin strain V1 genome by shotgun sequencing: a resource for DNA microarrays and expression profiling. Mol Biochem Parasitol 2001; 113(2): 337-40.

[25] Saxena A, Lahav T, Holland N, et al. Analysis of the Leishmania donovani transcriptome reveals an ordered progression of transient and permanent changes in gene expression during differentiation. Mol Biochem Parasitol 2007; 152(1): 53-65.

[26] Akopyants NS, Matlib RS, Bukanova EN, et al. Expression profiling using random genomic DNA microarrays identifies differentially expressed genes associated with three major developmental stages of the protozoan parasite Leishmania major. Mol Biochem Parasitol 2004; 136(1): 71-86.

[27] Clayton C, Shapira M. Post-transcriptional regulation of gene expression in trypanosomes and leishmanias. Mol Biochem Parasitol 2007; 156(2): 93-101.

[28] Greenbaum D, Colangelo C, Williams K, Gerstein M. Comparing protein abundance and mRNA expression levels on a genomic scale. Genome Biol 2003; 4(9): 117.

[29] Selvapandiyan A, Duncan R, Debrabant A, et al. Genetically modified live attenuated parasites as vaccines for leishmaniasis. Indian J Med Res 2006; 123(3): 455-66.

[30] Amaral VF, Teva A, Oliveira-Neto MP, et al. Study of the safety, immunogenicity and efficacy of attenuated and killed Leishmania (Leishmania) major vaccines in a rhesus monkey (Macaca mulatta) model of the human disease. Mem Inst Oswaldo Cruz 2002; 97(7): 1041-8.

[31] Spath GF, Lye LF, Segawa H, Turco SJ, Beverley SM. Identification of a compensatory mutant (lpg2-REV) of Leishmania major able to survive as amastigotes within macrophages without LPG2-dependent glycoconjugates and its significance to virulence and immunization strategies. Infect Immun 2004; 72(6): 3622-7.

[32] Mori K, Yasutomi Y, Ohgimoto S, et al. Quintuple deglycosylation mutant of simian immunodeficiency virus SIVmac239 in rhesus macaques: robust primary replication, tightly contained chronic infection, and elicitation of potent immunity against the parental wild-type strain. J Virol 2001; 75(9): 4023-8.

[33] Parkin NT, Chiu P, Coelingh K. Genetically engineered live attenuated influenza A virus vaccine candidates. J Virol 1997; 71(4): $2772-8$ (http://creativecommons.org/licenses/by-nc/3.0/) which permits unrestricted, non-commercial use, distribution and reproduction in any medium, provided the work is properly cited. 